\title{
Item distribution and inter-rater reliability of the German version of the quality of life in Alzheimer's disease scale (QoL-AD) proxy for people with dementia living in nursing homes
}

\author{
Martin Nikolaus Dichter ${ }^{1,2^{*}}$ (D), Eva-Maria Wolschon ${ }^{3}$, Christian G. G. Schwab ${ }^{1,2}$, Gabriele Meyer ${ }^{4}$ and Sascha Köpke ${ }^{3}$
}

\begin{abstract}
Background: The Quality of Life in Alzheimer's disease scale (QoL-AD) is a widely used Health Related Quality of Life (HRQOL) instrument. However, studies investigating the instrument's inter-rater reliability (IRR) are missing. This study aimed to determine the item distribution and IRR of the German proxy version of the QoL-AD (13 Items) and a nursing home-specific instrument version (QoL-AD NH, 15 Items).

Methods: The instruments were applied to 73 people with dementia living in eight nursing homes in Germany. Individuals with dementia were assessed two times by blinded proxy raters. The IRR analyses were based on methodological criteria of the quality appraisal tool for studies of diagnostic reliability (QAREL), the COSMIN group and the single-measure Intra-Class Correlation Coefficient (ICC) for absolute agreement $\geq 0.70$.

Results: All items for both instrument versions demonstrated acceptable item difficulty, with the exception of one item (QoL-AD proxy). The IRR was moderate for the QoL-AD (ICC: 0.65) and insufficient for the QoL-AD $\mathrm{NH}$ (ICC: 0.18). The additional computation of the average measure ICC for two proxy-raters demonstrated a strong IRR (ICC: 0.79) for the QOL-AD and a weak IRR for the QoL-AD NH (ICC: 0.31). The detailed analysis of the IRR for each item underpinned the need for the further development of both instruments.

Conclusions: The unsatisfactory IRRs for both instruments highlight the need for the development of a user guide including general instructions for instrument application as well as definitions and examples reflecting item meaning. Priority should be given to the development of reliable proxy-person versions of both instruments.
\end{abstract}

Trial registration: ClinicalTrials.gov: NCT02295462, Date of registration: 11-20-2014.

Keywords: Quality of life, Nursing homes, Dementia, Psychometric properties, Reliability

\section{Background}

Health Related Quality of Life (HRQoL) has become an important outcome in dementia research $[1,2]$. HRQoL is defined as a "multidimensional concept that reflects the individual's subjective perception of the impact of a health

\footnotetext{
* Correspondence: Martin.Dichter@dzne.de

1 German Center for Neurodegenerative Diseases (DZNE), Stockumer Straße 12, 58453 Witten, Germany

${ }^{2}$ School of Nursing Science, Witten/Herdecke University, Stockumer Straße

12, 58453 Witten, Germany

Full list of author information is available at the end of the article
}

condition on everyday living" [3]. The Quality of life in Alzheimer's Disease (QoL-AD) scale has been widely used to measure HRQoL in people with dementia [4-7]. The QoL-AD has been developed in the US [4] and is based on the conceptual work by Lawton [8] who defines the QoL concept as multidimensional, including subjective (e.g., perceived QoL and psychological well-being) and objective components (e.g., behavioral competence and environment). The instrument had originally been developed as self-rating version for community-dwelling people with

(c) The Author(s). 2018 Open Access This article is distributed under the terms of the Creative Commons Attribution 4.0 International License (http://creativecommons.org/licenses/by/4.0/), which permits unrestricted use, distribution, and reproduction in any medium, provided you give appropriate credit to the original author(s) and the source, provide a link to the Creative Commons license, and indicate if changes were made. The Creative Commons Public Domain Dedication waiver (http://creativecommons.org/publicdomain/zero/1.0/) applies to the data made available in this article, unless otherwise stated. 
dementia but has also been used frequently as proxy-instrument in the nursing home setting [9-11].

In addition to the self- and proxy version of the QoL-AD, Edelman et al. [5] have developed the QoL-AD $\mathrm{NH}$, an adapted version of the original instrument particularly for people living in nursing homes. Whereas self-rating means that the QoL of a person with dementia is rated by herself/himself, a proxy-rating is defined as the QoL rating of a particular person with dementia by a proxy e.g. relative or caregiver of the person with dementia. According to Pickard \& Knight [12] the proxy-rating perspectives "proxy-proxy" and "proxy-person" have been distinguished. While in the former perspective the proxy rates the HRQoL of a person with dementia from his/her proxy perspective, in the latter perspective a proxy assesses the QoL of a person with dementia as he/she thinks the person with dementia would rate him or herself [12]. Both proxy perspectives are appropriate for the assessment of HRQoL. Unfortunately, the applied proxy perspective mostly remains unclear in the literature [13]. One recent study investigated both perspectives (proxy-proxy and proxy-person), comparing them to self-reports of people with and without dementia in nursing homes. The three perspectives were assessed with different versions of the EuroQol-5D [13]. The results show that both proxy-perspectives overestimate low self-reports and underestimate high self-reports. The tendency to attenuate self-ratings existed for both proxy-perspectives with a smaller perspective gap between self-ratings and the proxy-person perspective [13]. These results highlight the need for a clear definition and description and the psychometric investigation of both proxy perspectives. In general, there is little information on the psychometric properties of the proxy versions of the QoL-AD and QoL-AD NH [1, 2]. In particular, there is a lack of information about the inter-rater reliability (IRR) of the QoL-AD proxy and QoL-AD NH proxy scales [2]. This lack of information is often neglected in the literature $[1,14-18]$ and its impact on the validity of the QoL-AD proxy and QoL-AD NH proxy scales is unclear [19].

A detailed user guide with instructions for the instrument application is available for the self-rating versions, but not for proxy-rating versions. It is particularly unclear whether the items of both proxy versions have to be rated from a proxy-proxy or a proxy-person perspective.

QoL-AD proxy and QoL-AD NH proxy are frequently applied which is most likely due to the instruments' anticipated feasibility. Thus, the low number of items (QoL-AD proxy=13 Items, QoL-AD NH proxy $=15$ Items) and the fact that no comprehensive training is necessary (instructions for the proxy versions are not available) allows a resource-saving data collection in contrast to other QoL measures for people with dementia (e.g. QUALIDEM, Dementia Care Mapping instrument). The number of missing values has been described as low $[9,11,20]$.

The discrepancy between this lack of knowledge and the uncontrolled usage of the QoL-AD proxy and QoL-AD NH proxy emphasizes the relevance of a comprehensive evaluation of the IRR of both measures.

While the German version of the QoL-AD proxy has been available for some years, we have only recently conducted a cross-cultural adaption of the QoL-AD NH proxy to the German context [21].

Based on the international lack of knowledge concerning the IRR of the QoL-AD proxy and QoL-AD NH proxy as well as different perspective gaps between self-ratings and proxy perspectives the objective of the present study was to evaluate item distribution and IRR of both instruments, based on a proxy-proxy perspective for the QoL-AD proxy and a proxy-person perspective for the QoL-AD NH proxy.

\section{Methods \\ Study design}

This study was conducted between June 2015 and March 2016 as a nested cohort study within the randomized controlled trial EPCentCare [22], which aimed to reduce antipsychotic medication in nursing home residents. EPCentCare was carried out in three German regions, whereas the present evaluation took place in Northern Germany only. The study sample consisted of people with dementia from eight nursing homes located in Schleswig-Holstein and Hamburg. The investigation of the IRR of the QoL-AD proxy and QoL-AD NH proxy was based on the criteria of the quality appraisal tool for studies of diagnostic reliability (QAREL) [23] and the COSMIN group [24] (e.g. sample size calculation, description of blinding of raters, description of raters).

\section{Sample size calculation}

The sample size calculation was based on an estimated intra-class correlation coefficient (ICC) of 0.75 , ratings of two independent raters (registered nurses and nursing assistants) and a width of 0.20 for the $95 \%$ Confidence Interval (CI). This resulted in a calculated sample of 75 residents with dementia [25].

\section{Procedures}

According to the inclusion criteria of the EPCentCare trial, nursing homes with at least 50 residents were eligible for participation in the study. For this IRR evaluation the predefined inclusion criteria for residents with dementia was a Dementia Screening Scale score $\geq 3$ [26]. Exclusion criteria were a temporary stay in respite care or a primary diagnosis of schizophrenia or bipolar disorders. 
Inclusion criteria for caregivers were at least half-time work and at least one year nursing training ("nursing assistant" qualification). Qualification levels of proxy-raters depended on organizational conditions and staffing levels at the time of data collection in the participating nursing homes. Additionally, caregivers had to have been at work on at least seven days within the last two weeks prior to data collection and had to have a close relationship with the assessed resident. Based on these criteria, caregivers were identified and assigned to the assessed residents by the management staff (head nurse) of each participating nursing home. The close relationship with the assessed resident enabled the caregivers to rate the HRQoL based on a proxy-person and proxy-proxy perspective.

The IRR evaluation was based on independent proxy-ratings from registered nurses or nursing assistants referring to the preceding two weeks. Both independent ratings for one measure and one resident took place in the same shift and under the same circumstances. Each caregiver was blinded to the ratings of the other rater. To ensure blinding of raters and standardized data collection, application of QoL-AD proxy and QoL-AD NH proxy was supervised by researchers of the EPcentCare trial, who had been trained in applying QoL instruments, prior to the data collection. The supervision included a short instruction about the assessed construct (e.g. HRQoL, agitated behavior), the underlying time frame (preceding two weeks) and the perspective (e.g. proxy-person). Based on different organizational conditions and staffing levels at the time of data collection, independent ratings by different qualified caregivers (registered nurses and nursing assistants) were possible. During the data collection occasion one caregiver assessed one QoL-AD proxy version at the beginning and one at the end of the data collection. The order of the QoL-AD proxy application was randomly applied.

\section{Instruments}

The QoL-AD proxy consists of 13 items which can be answered by self-rating (person with dementia) or proxy-rating (e.g. caregiver). Response options for all items are "poor", "fair", "good" and "excellent" resulting in item scores between 1 to 4 and total scores between 13 and 52, with higher scores indicate higher HRQoL. In 2005 the QoL-AD was translated into German language [27]. For this investigation the German version provided by Mapi Research Trust, Lyon was used [28]. Although information related to the linguistic validation of the German version is not available, in two recent studies, the German QoL-AD proxy demonstrated sufficient internal consistency and structural validity $[6,29]$.

For the nursing home version (QoL-AD NH proxy), two items of the original version concerning financial and marital status (Money, Marriage) were removed and four items added (People who work here, Ability to take care of oneself, Ability to live with others, and Ability to make choices in one's life) [5]. The response options for the QoL-AD NH correspond to the original version, resulting in total scores from 15 to 60. In 2016 the nursing home version was translated into German and linguistically validated [21]. While no information on psychometric properties are available for the German version, the original US proxy-proxy version showed sufficient internal consistency in three studies $[5,30,31]$ and a nearly perfect IRR [31].

Cognitive impairment of participating residents was assessed by nursing staff using the Dementia Screening Scale (DSS) [26], a seven item measure with a three point response scale $(0,1,2)$ resulting in scores between 0 and 14 , with higher scores indicating more cognitive impairment.

Agitated behavior was assessed by nursing staff using a adapted German version [32] of the Cohen-Mansfield Agitation Inventory (CMAI) [33, 34], a proxy-measurement consisting of 25 items rated on a seven-point scale of frequency of occurrence resulting in scores between 25 and 175 [35], with higher scores indicating higher frequencies of agitation. Age, gender, length of stay in nursing home in months, and care dependency level (0, I, II, or III) as defined by the German long-term care insurance, were taken from the residents' case files. In addition, proxy-rater characteristics were assessed with single items.

\section{Data analysis}

Sample characteristics and item distribution were computed using descriptive statistics. Based on the item distribution the item difficulty was proven. An item mean in the first $20 \%$ of the scale was defined as floor effect and in the last $20 \%$ as ceiling effect. To gain comprehensive information on the degree of IRR for the QoL-AD proxy and QoL-AD NH proxy, a reliability coefficient was calculated for each measurement item and all measurement items in total. This procedure was based on earlier IRR studies and allowed a detailed interpretation and comparison of the IRR of each item [36, 37]. The IRR for each item was based on a calculation of the overall proportion of agreement $\left(\mathrm{p}_{\mathrm{o}}\right)$. Moreover, we computed the linear weighted $\kappa$ statistics for ordinal data $\left(\kappa_{\mathrm{w}}\right)[38,39]$, because $\mathrm{p}_{\mathrm{o}}$ ignores the possibility that agreement could occur only by chance and instead considers only crude agreement. The two paradoxical properties of $\mathrm{K}$ statistics were considered during the interpretation of the results [40]. The interpretation of $\kappa_{\mathrm{w}}$ values was based on the following ranges: $0.00-0.20$, slight; $0.21-0.40$, fair; $0.41-0.60$, moderate; 0.61-0.80, substantial; and 0.81-1.00, nearly perfect [41]. The IRR of the QoL-AD proxy and QoL-AD NH proxy in total 
were evaluated using Intraclass Correlation Coefficients (ICC) based on a two-way random-effects model for absolute agreement. Additionally, the average-measure ICC for two raters was calculated. This coefficient estimates the IRR of a collaborative QoL-AD proxy respectively QoL-AD NH proxy rating by two raters. Based on the recommendation by Terwee et al. [24], we targeted $\kappa_{w}$ and ICC values $\geq 0.7$. To analyze the level of uncertainty, 95\% CIs for ICCs and $\kappa_{w}$ values were examined. The 95\% CI for $\kappa_{\mathrm{w}}$ values was based on 10,000 bootstrapped samples and determined using the percentile method [42], which included using the 0.025 and 0.975 percentile levels of the estimated Kappa distributions as interval limits. Statistical analysis was performed using R Version 3.2.4 [43] and the software packages "irr" Version 0.84 [44] and "boot" Version 1.3-18 [45, 46].

\section{Results}

\section{Characteristics of the sample}

The sample consisted of 73 residents with dementia and 21 caregivers from eight nursing homes (Table 1).

\section{Item distribution}

The descriptive investigation of the QoL-AD proxy items (proxy-proxy perspective) demonstrated a balanced distribution (Table 2). The response option "good" was used most frequently whereas the response category "excellent" was used least often. Distributions of the other response options were balanced. Only one item showed a floor effect (item 10, ability to do chores around the house).

Analyses for the QoL-AD NH proxy (proxy-person perspective) yielded similar results. However, no item showed floor or ceiling effects.

Missing value analyses demonstrated low percentages of missing values in general. Only item 12 (Money) of the QoL-AD proxy showed a high percentage of missing values $(29 \%)$. The main reason for this was nurses' refusal to rate or a lack of knowledge to assess residents' financial situation. A descriptive comparison of the elven comparable items shows no clear pattern that one perspective or QoL scale lead to higher QoL ratings.

\section{Inter-rater reliability}

The results of the IRR evaluation for the QoL-AD proxy and the QoL-AD NH proxy are displayed in Table 3. Based on the high percentage of missing values for item 12 of the QoL-AD proxy two ICC values based on different sample were computed. Both resulting ICC values (ICC: $0.65=13$ items, ICC: $0.63=12$ items) demonstrated a moderate IRR for the QoL-AD proxy. The average-measure ICC for two proxy-raters demonstrated a strong IRR of 0.79 (13 items) or 0.77 (12 items) for the QoL-AD proxy. In contrast, the IRR results for the
Table 1 Characteristics of the sample

\begin{tabular}{|c|c|c|}
\hline \multicolumn{3}{|l|}{ Characteristics } \\
\hline People with dementia & $\mathrm{n}$ & $=73$ \\
\hline Age in years ${ }^{a}$ & 87.38 & $( \pm 7.71)$ \\
\hline Female $^{a}$ & 38 & $(71.7 \%)$ \\
\hline Length of stay in nursing home in months ${ }^{a}$ & 34.57 & $( \pm 31.14)$ \\
\hline \multicolumn{3}{|l|}{ Care dependency levels $s^{\mathrm{a}, \mathrm{b}}$} \\
\hline - None & 0 & $(0.0 \%)$ \\
\hline - considerable (= I) & 9 & $(17.0 \%)$ \\
\hline - severe $(=\|)$ & 25 & $(47.2 \%)$ \\
\hline - most severe $(\geq I I)$ & 19 & $(35.8 \%)$ \\
\hline Dementia Screening Scale (DSS) & 9.01 & $( \pm 3.14)$ \\
\hline • mild or moderate dementia (3-7) & 28 & (38.4\%) \\
\hline • severe dementia (8-14) & 45 & $(61.6 \%)$ \\
\hline Challenging behaviour $(\mathrm{CMAI})^{\mathrm{a}}$ & 34.87 & $( \pm 10.48)$ \\
\hline Caregivers (proxy-raters) & $\mathrm{n}$ & $=8^{c}$ \\
\hline Age in years & 34.75 & $( \pm 13.76)$ \\
\hline Female & 8 & $(100.0 \%)$ \\
\hline Registered nurses & 5 & $(62.5 \%)$ \\
\hline Working experience in years & 9.38 & $( \pm 5.63)$ \\
\hline Working hours per week & 38.75 & $( \pm 3.54)$ \\
\hline \multicolumn{3}{|l|}{ Experience in proxy-ratings } \\
\hline$\cdot$ no & 6 & $(75.0 \%)$ \\
\hline - yes, at least once & 1 & $(12.5 \%)$ \\
\hline - yes, regularly ( $\geq$ twice a month) & 1 & $(12.5 \%)$ \\
\hline
\end{tabular}

Data are reported as means $( \pm \mathrm{SD})$ or absolute number (\%)

These results were based on $n=53$ people with dementia, because these characteristics were missing for $n=20$ people of the total sample

${ }^{\mathrm{b}}$ As determined by expert raters of the medical service of the statutory longterm care insurance system

'The analyzed data includes answers of 21 raters. For 13 proxy-raters there are no data available

Qol-AD NH proxy were weak: $(0.18$ or 0.31 for the average-measure ICC).

For the QoL-AD proxy, $\kappa_{\mathrm{w}}$ values ranged from 0.07 (item 12: money) to 0.55 (item 6: family). The $\mathrm{p}_{\mathrm{o}}$ ranged between 0.33 (item 12: money) and 0.63 (item 13: life as a whole). IRR results for the QoL-AD NH proxy items ranged between -0.03 (item 4: living situation) to 0.38 (item 15: live overall) for the $\kappa_{\mathrm{w}}$ and between 0.29 (item 4: living situation) to 0.55 (item 15: live overall) for the $\mathrm{p}_{\mathrm{o}}$.

\section{Discussion}

\section{Item distribution}

This study describes a comprehensive evaluation of the item distribution and inter-rater reliability of the German QoL-AD proxy and QoL-AD NH proxy.

The investigation of the item distribution of the QoL-AD proxy demonstrated a balanced distribution of the four response options. Twelve out of 13 items showed an acceptable item difficulty. Only one item 


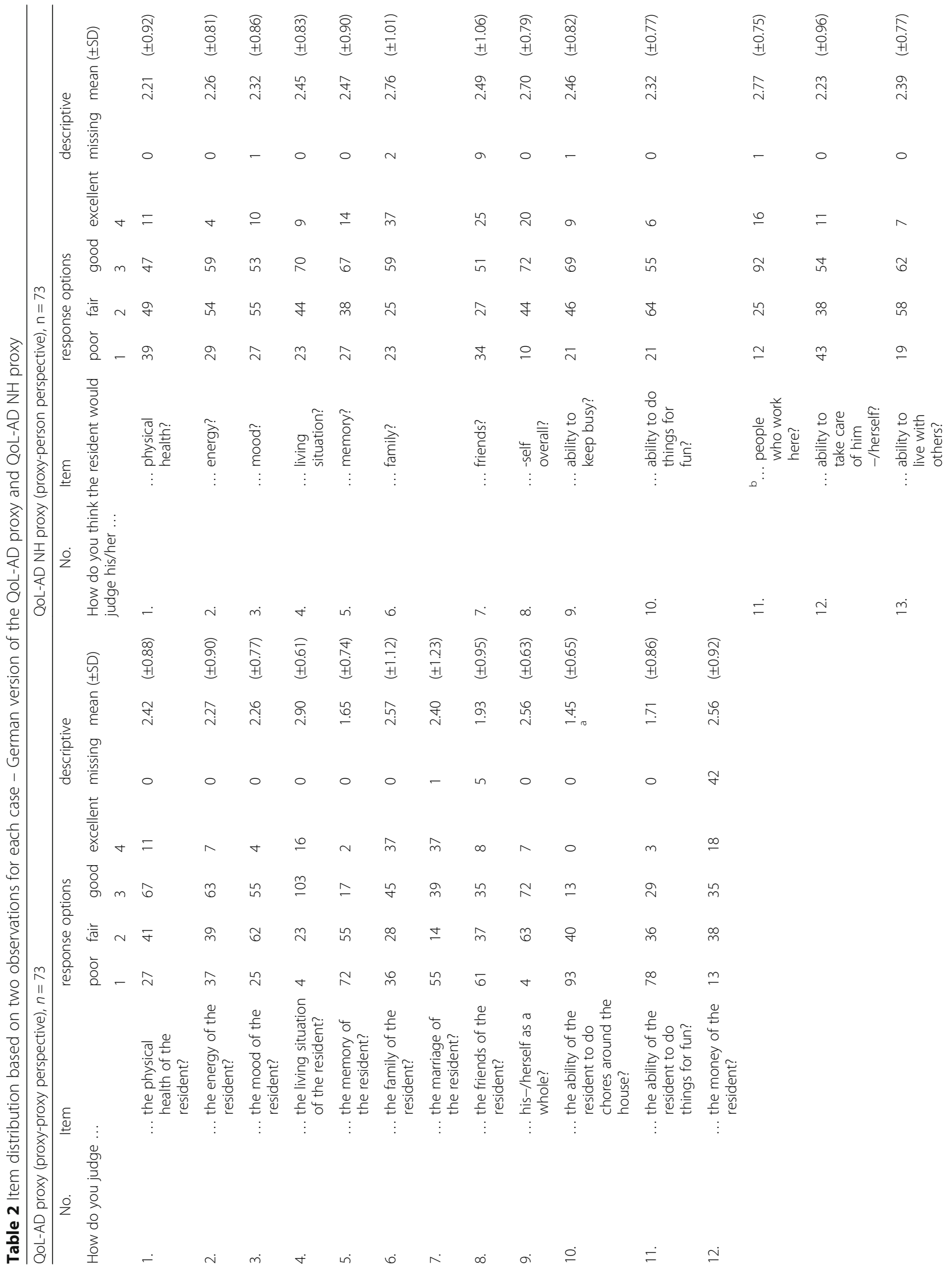




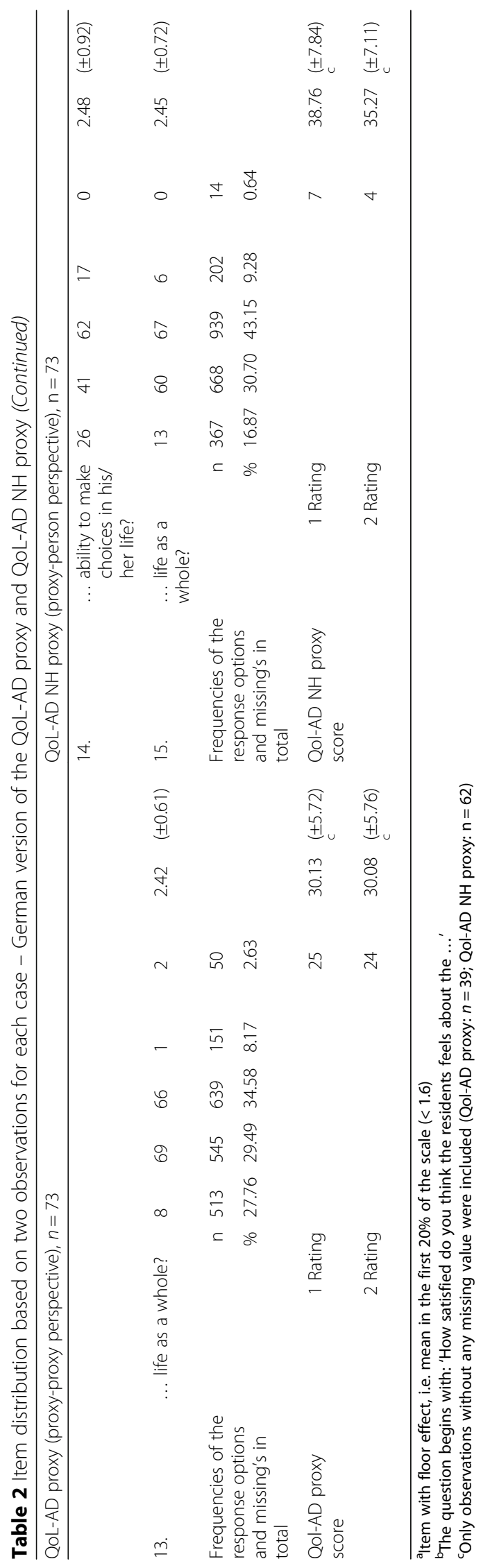




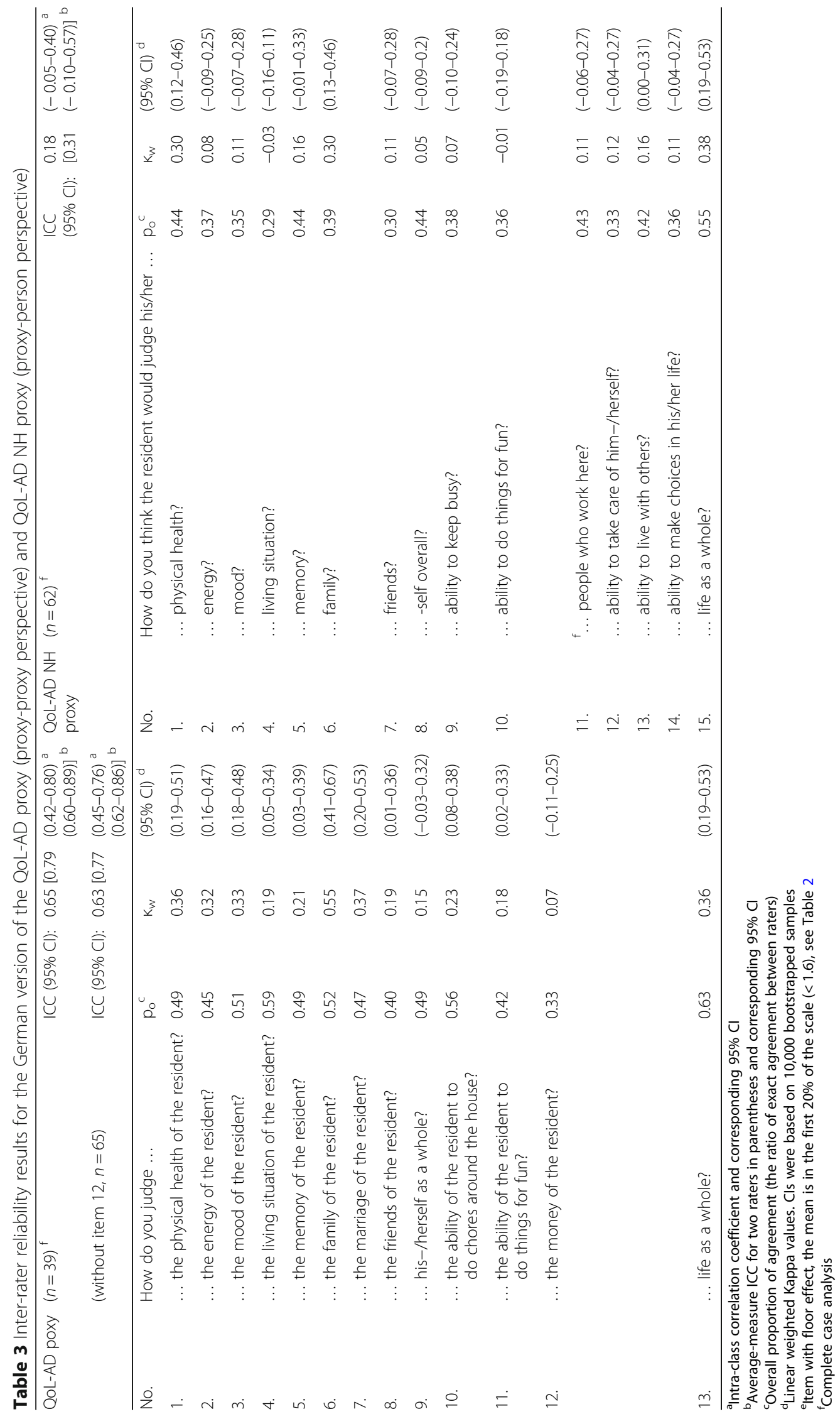


(item 10: ability to do chores around the house) showed a floor effect and item 12 (Money) showed a high percentage of missing values (29\%). One reason for these results for item 10 and 12 might be a missing cross-cultural adaption of the QoL-AD proxy for the German context and in particular for German nursing homes.

The analysis for the QoL-AD NH proxy yielded similar results with an acceptable item difficulty for all 15 items. With the exception of the identified floor effect for item 10 of the QoL-AD proxy, these descriptive results are in the line with previous results $[4,5,21,47]$. Given the relatively small sample size, the identified floor effect for the item 10 of the QoL-AD proxy must be interpreted with caution.

A descriptive comparison of the eleven comparable items shows no clear pattern that one perspective leads to higher QoL ratings. Thus, compared to the comparable items rated in both perspectives, we identified higher mean values for the items 1,2 and 4 of the QoL-AD (proxy-proxy) and for the items 3, 5, 6, 7, 8, 9, 10 and 15 of the QoL-AD NH (proxy-person). We assumed systematically higher QoL ratings based on a proxy-person perspective compared to a proxy-proxy perspective. This assumption was based on previous studies, which demonstrated systematically lower proxy-based QoL ratings compared to self-ratings $[48,49]$, and on one recent study, which showed a smaller perspective gap between self-ratings and the proxy-person perspective [13]. The major reason for the missing difference between both perspectives might be the only moderate to weak IRR of the applied scales and perspectives.

\section{Inter-rater reliability}

The IRR results demonstrate a moderate IRR for the QoL-AD proxy (ICC: $0.65,13$ items, ICC: $0.63,12$ items) and an insufficient IRR for the QOL-AD NH (ICC: 0.18 ). The additional computation of the average measure ICC for two proxy-raters demonstrated a strong IRR of 0.79 (13 items) or 0.77 (12 items) for the Qol-AD proxy and a weak ICC for the QoL-AD NH proxy (0.31).

The detailed analysis of the IRR of each item yielded heterogeneous results. Based on $\mathrm{k}_{\mathrm{w}}$ and $\mathrm{p}_{\mathrm{o}}$, only item 6 (family) of the QoL-AD proxy demonstrated a moderate IRR. All other QoL-AD proxy items showed fair (items 1: physical health, 2: energy, 3: mood, 5: memory, 7: marriage, 10: ability to do chores around the house, 13: life as a whole) or slight IRR (items: 4: living situation, 8: friends, 9: his-/herself as a whole, 11: ability to do things for fun, 12: money). Based on the high number of missing values and the slight IRR of item 12 (money of the resident) we recommend the exclusion of this item for the QoL-AD proxy application in nursing homes.
For the QoL-AD NH proxy only items 1 (physical health) and 15 (life overall) yielded a fair IRR. All other items demonstrated a slight IRR.

The in-depth analysis of the IRR indicates the need for improvement of both instruments for their application in research and practice. An improvement of the IRR might be reached through a structured instrument user guide including clear definitions and examples related to the meaning of each QoL-AD proxy and QoL-AD NH proxy item. The positive effect of such a user guide has been demonstrated in a recent IRR study on the dementia-specific QoL instrument QUALIDEM [37].

This study is the first IRR study dealing with the proxy version of the QoL-AD. Therefore, study results can only be compared to the IRR results of other dementia-specific QoL and HRQoL instruments [2]. The IRR of the Alzheimer Disease-Related Quality of Life (ADRQL) instrument was evaluated in two studies showing different results. While the US version of the ADRQL demonstrated excellent IRR with ICC values between 0.90 and 1.0 [31], a Swiss study identified slight to moderate ICC values depending on ADRQL subscales (Social interaction: $\mathrm{ICC}=0.36$, Awareness of self, ICC: 0.61, Feelings and mood, ICC: 0.13 . Enjoyment of activities, ICC: 0.02, Response to surrounding,: ICC: - 0.17) [50]. Both study results have to be interpreted with caution because of several methodological limitations [2]. The Quality of Life in Late stage Dementia scale (QUALID) demonstrated good IRR with an ICC value of 0.83 [51]. For the instrument QUALIDEM a recent study yielded a good IRR for all subscales for people with mild to very severe dementia (ICC $=0.79-0.96$ ) with the exception of the subscale negative affect $(\mathrm{ICC}=0.64)$ [37]. These three instruments reflect overall QoL. One frequently used instrument for the assessment of dementia-specific HRQoL is the DEMQOL [17]. Unfortunately, no information were available on IRR of the DEMQOL proxy-version which would have allowed a comparison to our study results [2]. Due to the heterogeneous range of QoL domains assessed with different QoL and HRQoL instruments, a comparison of IRR results between different instruments is limited. However, IRR results of instruments like QUALIDEM and QUALID demonstrate that good IRR values are achievable for proxy-rated dementia-specific QoL.

Our IRR results for the QoL-AD NH proxy can be compared to one previous US study which demonstrated a high IRR with a ICC value of 0.99 [31]. In contrast, our IRR results seem low. However, the study by Sloane et al. [31] had several methodological limitations and a proxy-proxy perspective. In contrast, our results are based on a proxy-person perspective. The different perspectives also explain the differences between the Qol-AD proxy and the QoL-AD NH proxy in our study. 
A comparison of equal items (item 1-6 and 7-10) from the QoL-AD proxy and QoL-AD NH proxy show lower $\mathrm{p}_{\mathrm{o}}$. and $\kappa_{\mathrm{w}}$ for the items assessed with the proxy-person perspective (QoL-AD-NH proxy).

The QoL-AD proxy data were based on a proxy-proxy perspective which means that proxies rate the HRQoL of care recipients from their proxy perspective. This might be an easier perspective compared to the so-called proxy-person perspective as there is no need for a perspective shift by the proxy rater. Nevertheless, proxy-proxy ratings can also be influenced by attitudes [52], life satisfaction, the assessment circumstances and challenging behaviors of people with dementia living in nursing homes [53]. Moreover, HRQoL ratings based on proxy-proxy ratings might not be particularly valid due to the partial loss of subjectivity of the HRQoL assessment [54].

In contrast, the proxy-person perspective requires a change of perspectives. Here, a proxy assesses the HRQoL of a person with dementia as he/she thinks the person with dementia would rate him or herself. It can be assumed that this perspective is more difficult for proxies, thus the required level of individuality for each HRQoL rating is high.

The results of a recent study [13], which showed a smaller perspective gap between self-ratings and the proxy-person perspective, underpin the need for the further development of the proxy-versions of the QoL-AD and QoL-AD NH to enable the assessment of an IRR proxy-person perspective for both instruments.

\section{Limitations}

This study is the first investigation of IRR of the QoL-AD based on a proxy-proxy perspective and the QoL-AD NH based on a proxy-person perspective. The strengths of this IRR study are the in-depth analysis of the IRR of each instrument item, the inclusion of people at all stages of dementia and the methodological rigor based on the criteria of the quality appraisal tool for studies of diagnostic reliability (QAREL) as well as the COSMIN group [24]. The following limitations should be considered when interpreting the results.

First, although the preplanned sample sizes were almost achieved, the identified width of CIs of the computed ICC values range between 0.29 and 0.67. Especially when interpreting the IRR of the QoL-AD NH this statistical uncertainty has to be taken into account. Our results provide a good basis for sample size calculations of further IRR studies.

Second, the applied data collection procedure led to a QoL-AD proxy and a QoL-AD NH proxy assessment for a resident with dementia by one caregiver on one occasion. Despite the random order of the ratings a possible influence of the rating of one measure on the rating of the second measure cannot be excluded.
Third, proxy-raters' characteristics have to be interpreted with caution due to the high rate of missing values.

Fourth, a close relationship between the proxy and the assessed person with dementia will help proxy raters to reach this required level of individuality. Usually primary models of nursing care are used in German nursing homes. The head nurse was instructed to assign a primary nurse to the assessed residents. Unfortunately, the shortage of nurses in German nursing homes may have influenced the assignment of the head nurses. A varying understanding of this criterion by the assigning head nurse may be jointly responsible for the IRR results.

\section{Conclusions}

This IRR study demonstrated a moderate IRR for the QoL-AD based on a proxy-proxy perspective and an insufficient IRR for the QoL-AD NH (proxy-person perspective). According to established cut off points for the interpretation of IRR values there is a need for the improvement of both instruments. We recommend the development of a user guide including general instructions for the application of both instruments as well as definitions and examples reflecting the meaning of each item. Priority should be given to the development of reliable proxy-person versions of both instruments. Until a user guide is available, the QoL-AD proxy might be conducted as a collaborative rating by at least two proxy-raters. However, this approach is limited to a proxy-proxy perspective which means partial loss of the subjectivity of the HRQoL assessment and as a result, a reduction in validity. Additionally, we recommend the exclusion of item 12 (money of the resident) for the QoL-AD proxy application in nursing homes.

The QoL-AD NH based on a proxy-person perspective should not be used until a user guide is available and further IRR studies have demonstrated an improved IRR.

\section{Abbreviations \\ 95\% Cl: 95\% Confidence Interval; ADRQL: Alzheimer Disease-Related Quality of Life; CMAl: Cohen-Mansfield Agitation Inventory; COSMIN: COnsensus- based Standards for the selection of health Measurement Instruments; DSS: Dementia Screening Scale; EPCentCare: Effect of person-centred care on antipsychotic drug use in nursing homes; HRQoL: Health Related Quality of Life; ICC: Intra-class correlation coefficient; IRR: Inter-rater reliability; $p_{0}$ : proportion of agreement; QAREL: Quality Appraisal tool for studies of diagnostic RELiability; QoL-AD NH: Quality of life in Alzheimer's Disease Nursing Home; QoL: Quality of Life; QoL-AD: Quality of life in Alzheimer's Disease; QUALID: Quality of Life in Late stage Dementia scale; $\mathrm{K}_{w}$ : weighted kappa statistics}

\section{Acknowledgements}

We would like to thank the caregivers and the people with dementia for their participation in our investigation.

\section{Funding}

German Federal Ministry of Education and Research (BMBF grant 01GY1335B). The funding body. The funders had no role in the design of the study, data collection, analysis or interpretation of the data. 


\section{Availability of data and materials}

All of the data necessary for a meta-analysis of inter-rater reliability of QoL$A D$ items are contained within the manuscript and its supplementary files. The datasets used and/or analyzed during the current study are available from the corresponding author on reasonable request.

\section{Authors' contributions}

MND, EMW, GM, and SK designed the study and developed the research question. EMW and SK performed the data collection. CGGS and MND carried out the data analysis. MND was responsible for the preparation of a first manuscript draft; all authors (MND, EMW, CGGS, SK, and GM) contributed to the manuscript preparation. All authors have read and approved the final version of the manuscript.

\section{Ethics approval and consent to participate}

Ethical approval was obtained from the ethics committee of the University of Lübeck (Ref. 14-239). Written information about the study was given to residents and residents' legal representatives, and written informed consent was obtained from residents or their legal representatives. Caregivers received written and oral information about the study before data collection. Voluntary participation in the data collection was considered as informed consent from caregivers. A written consent from the caregivers was waived by the ethics committee.

\section{Consent for publication}

Not applicable.

\section{Competing interests}

All authors declare that they have no competing interests. GM is a member of the BMC Geriatrics editorial board, but was not involved in the review process.

\section{Publisher's Note}

Springer Nature remains neutral with regard to jurisdictional claims in published maps and institutional affiliations.

\section{Author details}

${ }^{1}$ German Center for Neurodegenerative Diseases (DZNE), Stockumer Straße 12, 58453 Witten, Germany. ${ }^{2}$ School of Nursing Science, Witten/Herdecke University, Stockumer Straße 12, 58453 Witten, Germany. ${ }^{3}$ Institute of Social Medicine and Epidemiology, University of Lübeck, Ratzeburger Allee 160, 23562 Lübeck, Germany. ${ }^{4}$ Institute for Health and Nursing Science, Medical Faculty, Martin Luther University, Halle-, Wittenberg, Germany.

\section{Received: 1 May 2017 Accepted: 10 June 2018}

\section{Published online: 19 June 2018}

\section{References}

1. Bowling A, Rowe G, Adams S, Sands P, Samsi K, Crane M, Joly L, Manthorpe J. Quality of life in dementia: a systematically conducted narrative review of dementia-specific measurement scales. Aging Ment Health. 2015;19(1):13-31.

2. Dichter MN, Schwab CG, Meyer G, Bartholomeyczik S, Halek M. Linguistic validation and reliability properties are weak investigated of most dementiaspecific quality of life measurements-a systematic review. J Clin Epidemiol. 2016;70:233-45.

3. Bullinger M, Anderson R, Cella D, Aaronson N. Developing and evaluating cross-cultural instruments from minimum requirements to optimal models. Qual Life Res. 1993;2(6):451-9.

4. Logsdon RG, Gibbons LE, McCurry SM, Teri L. Quality of life in Alzheimer's disease: patient and caregiver reports. J Ment Health Aging. 1999;5(1):21-32.

5. Edelman P, Fulton BR, Kuhn D, Chang CH. A comparison of three methods of measuring dementia-specific quality of life: perspectives of residents, staff, and observers. Gerontologist. 2005;45:Spec No 1(1):27-36.

6. Hylla J, Schwab CGG, Isfort M, Halek M, Dichter MN. Internal consistency and construct validity of the quality of life in Alzheimer's disease (QoL-AD) proxy - a secondary data analysis. Pflege. 2016;29(4):183-91. https://doi.org/ 10.1024/1012-5302/a000xxx.

7. Barrios H, Verdelho A, Narciso S, Goncalves-Pereira M, Logsdon R, de Mendonca A. Quality of life in patients with cognitive impairment: validation of the quality of life-Alzheimer's disease scale in Portugal. Int Psychogeriatr. 2013;25(7):1085-96.

8. Lawton MP. A multidimensional view of quality of life in frail elders. In: Birren EJ, Lubben EJ, Rowe CJ, Deutchman ED, editors. The concept and measurement of quality of life in the frail elderly. San Diego: Academic Press; 1991. p. 3-23.

9. Dichter MN, Quasdorf T, Schwab CG, Trutschel D, Haastert B, Riesner C, Bartholomeyczik S, Halek M. Dementia care mapping: effects on residents' quality of life and challenging behavior in German nursing homes. A quasiexperimental trial. Int Psychogeriatr. 2015;27:1875-92.

10. Beerens HC, de Boer B, Zwakhalen SM, Tan FE, Ruwaard D, Hamers JP, et al. The association between aspects of daily life and quality of life of people with dementia living in long-term care facilities: a momentary assessment study. Int Psychogeriatr. 2016; 28(8):1323-31.

11. Nielsen AB, Siersma V, Waldemar G, Waldorff FB. Poor self-rated health did not increase risk of permanent nursing placement or mortality in people with mild Alzheimer's disease. BMC Geriatr. 2016; 16:87.

12. Pickard AS, Knight SJ. Proxy evaluation of health-related quality of life: a conceptual framework for understanding multiple proxy perspectives. Med Care. 2005;43(5):493-9.

13. Leontjevas R, Teerenstra S, Smalbrugge M, Koopmans RT, Gerritsen DL. Quality of life assessments in nursing homes revealed a tendency of proxies to moderate patients' self-reports. J Clin Epidemiol. 2016;80:123-33.

14. Perales J, Cosco TD, Stephan BC, Haro JM, Brayne C. Health-related qualityof-life instruments for Alzheimer's disease and mixed dementia. Int Psychogeriatrics. 2013;25(5):691-706.

15. Graske J, Fischer T, Kuhlmey A, Wolf-Ostermann K. Dementia-specific quality of life instruments and their appropriateness in shared-housing arrangements-a literature study. Geriatr Nurs. 2012;33(3):204-16.

16. Oppikofer S. Quality of life in dementia. Identification, review and documentation of existing instruments to measure quality of life in people with severe dementia. [Lebensqualität bei Demenz. Eine Bestandsaufnahme, Sichtung und Dokumentation bestehender Instrumente zur Messung der Lebensqualität bei Menschen mit schwerer Demenz]. In: Zürich UH, editor. Zürcher Schriften zur Gerontologie. Zürich: University Hospital Zürich; 2008.

17. Smith SC, Lamping DL, Banerjee S, Harwood RH, Foley B, Smith P, Cook JC, Murray J, Prince M, Levin E, et al. Measurement of health-related quality of life for people with dementia: development of a new instrument (DEMQOL) and an evaluation of current methodology. Health Technol Assess NHS R\&D HTA Programme. 2005;9(10):1-110.

18. Scholzel-Dorenbos CJ, van der Steen MJ, Engels LK, Olde Rikkert MG. Assessment of quality of life as outcome in dementia and $\mathrm{MCl}$ intervention trials: a systematic review. Alzheimer Dis Assoc Disord. 2007;21(2):172-8.

19. Dichter M. Inter-rater reliability of the Qol-measure QUALIDEM. BMC Nurs. 2015;14(Suppl1):12.

20. Beerens HC, Sutcliffe C, Renom-Guiteras A, Soto ME, Suhonen R, Zabalegu A, Bokberg C, Saks K, Hamers JP, RightTimePlaceCare C. Quality of life and quality of care for people with dementia receiving long term institutional care or professional home care: the European RightTimePlaceCare study. Am Med Dir Assoc. 2014;15(1):54-61.

21. Dichter MN, Wolschon EM, Meyer G, Köpke S. Cross-cultural adaptation of the German version of the quality of life in Alzheimer's disease scale nursing home version (QoL-AD NH). Int Psychogeriatr. 2016:28:1-2.

22. Richter C, Berg A, Fleischer S, Kopke S, Balzer K, Fick EM, Sonnichsen A, Loscher S, Vollmar HC, Haastert B, et al. Effect of person-centred care on antipsychotic drug use in nursing homes (EPCentCare): study protocol for a cluster-randomised controlled trial. Implementation science : IS. 2015;10:82.

23. Lucas NP, Macaskill P, Irwig L, Bogduk N. The development of a quality appraisal tool for studies of diagnostic reliability (QAREL). J Clin Epidemiol. 2010;63(8):854-61

24. Terwee CB, Bot SD, de Boer MR, van der Windt DA, Knol DL, Dekker J, Bouter LM, de Vet HC. Quality criteria were proposed for measurement properties of health status questionnaires. J Clin Epidemiol. 2007;60(1): 34-42.

25. Bonett DG. Sample size requirements for estimating intraclass correlations with desired precision. Stat Med. 2002;21(9):1331-5.

26. Köhler L, Weyerer $S$, Schäufele M. Proxy screening tools improve the recognition of dementia in old-age homes: results of a validation study. Age Ageing. 2007; 36(5):549-54.

27. Pantel J, Bockenheimer-Lucius G, Ebsen I, Müller R, Hustedt P, Diehm A. Psychopharmaka im Altenpflegeheim. Eine interdisziplinäre Untersuchung 
unter Berücksichtigung gerontopsychiatrischer, ethischer und juristischer Aspekte. Frankfurt am Main: BHF-Bank-Stiftung; 2006.

28. Mapi Research Trust: QoL-AD Germany. German Version of 13 Jun 13 - MAPI Institute. In. Lyon: Mapi Research Trust; 2013.

29. Gräske J, Verbeek H, Gellert P, Fischer T, Kuhlmey A, Wolf-Ostermann K. How to measure quality of life in shared-housing arrangements? A comparison of dementia-specific instruments. Qual Life Res. 2014;23(2):549-59.

30. Edelman $P$, Fulton BR, Kuhn D. Comparison of dementia-specific quality of life measures in adult day centers. Home Health Care Serv Q. 2004;23(1):25-42.

31. Sloane PD, Zimmerman S, Williams CS, Reed PS, Gill KS, Preisser JS. Evaluating the quality of life of long-term care residents with dementia. Gerontologist. 2005;45 Spec No 1(1):37-49.

32. Weyerer S, Schäufele M, Hendlmeier I, Kofahl C, Sattel H, B J h f n AUTOREN. Evaluation of dementia specific residential care in Hamburg [Evaluation der Besonderen Stationären Dementenbetreuung in Hamburg]. Mannheim: Zentralinstitut für Seelische Gesundheit: Mannheim; 2004.

33. Cohen-Mansfield J. Assessment of agitation. Int Psychogeriatr. 1996;8:233-45.

34. Cohen-Mansfield J, Marx MS, Rosenthal AS. A description of agitation in a nursing home. J Gerontol. 1989;44:M77-84.

35. Müller B, Staudinger B. Evaluation of a Multimodal Treatment Program for Agitated Behavior in Nursing Home Residents with Dementia [Wirksamkeit eines stationären multimodalen Behandlungspro-gramms bei agitierten Verhaltensweisen dementer Heimbewohner]. Zeitschrift für Pflegewissenschaft und psychische Gesundheit. 2010;4:1-9.

36. Dichter MN, Schwab C, Meyer G, Bartholomeyczik S, Dortmann O, Halek M. Measuring the quality of life in mild to very severe dementia: testing the inter-rater and intra-rater reliability of the German version of the QUALIDEM. Int Psychogeriatr. 2014;26(5):825-36.

37. Dichter MN, Schwab CG, Meyer G, Bartholomeyczik S, Halek M. Item distribution, internal consistency and inter-rater reliability of the German version of the QUALIDEM for people with mild to severe and very severe dementia. BMC Geriatr. 2016;16(126) https://doi.org/10.1186/s12877-1201610296-12870.

38. Cohen J. Weighted kappa: nominal scale agreement with provision for scaled disagreement or partial credit. Psychol Bull. 1968;70(4):213-20.

39. Wirtz M, Caspar F. Rater-agreement and Rater-reliability.Methods to determine and improve the reliability of ratings by category systems and rating scales Beurteilungsübereinstimmung und Beurteilerreliabilität. In: Methoden zur Bestimmung und Verbesserung der Zuverlässigkeit von Einschätzungen mittels Kategoriensystemen und Ratingskalen. Hogrefe Verlag: Göttingen; 2002.

40. Mayer H, Nonn C, Osterbrink J, Evers GC. Quality criteria of assessment scales - Cohen's kappa as measure of interrator reliability (1). Pflege. 2004; 17(1):36-46.

41. Landis JR, Koch GG. The measurement of observer agreement for categorical data. Biometrics. 1977;33(1):159-74.

42. Carpenter J, Bithell J. Bootstrap confidence intervals: when, which, what? A practical guide for medical statisticians. Stat Med. 2000;19(9):1141-64.

43. Core R. Team: R: A language and environment for statistical computing. Vienna: R Foundation for Statistical Computing; 2015.

44. Gamer M, Lemon J, Fellows I,PS. IRR: Various Coefficients of Interrater Reliability and Agreement. R package version 0.84. p. 2012. http://www.r-project.org.

45. Canty A, Ripley B. Boot: Bootstrap R (S-Plus) Functions. R package version 1. 3-16. p. 2015. http://www.r-project.org.

46. Davison AC, Hinkley DV. Bootstrap methods and their applications. Cambridge: Cambridge University Press; 1997.

47. Wolak A, Jolly D, Dramé M, Boyer F, Morrone I, Aquino JP, Rouaud O, Perret Guillaume C, Ravenel E, Dantoine T, et al. Quality of life in dementia: psychometric properties of a French language version of the dementia quality of life questionnaire (DQoL). European Geriatric Medicine. 2010;1(6): $334-47$

48. Gräske J, Fischer T, Kuhlmey A, Wolf-Ostermann K. Quality of life in dementia care - differences in quality of life measurements performed by residents with dementia and by nursing staff. Aging Ment Health. 2012; 16(7):819-27.

49. Jacob L, Han JW, Kim TH, Park JH, Lee SB, Lee JJ, et al. How Different are Quality of Life Ratings for People with Dementia Reported by Their Family Caregivers from Those Reported by the Patients Themselves? J Alzheimers Dis. 2017; 55(1):259-67.
50. Menzi-Kuhn C. [Quality of life of people with dementia in institutional care] Lebensqualität von Menschen mit Demenz in stationären Langzeitpflegeeinrichtungen. In: Unpublished Masterthesis. Maastricht: Maastricht University; 2006

51. Weiner MF, Martin-Cook K, Svetlik DA, Saine K, Foster B, Fontaine CS. The quality of life in late-stage dementia (QUALID) scale. J Am Med Dir Assoc. 2000;1(3):114-6.

52. Winzelberg GS, Williams CS, Preisser JS, Zimmerman S, Sloane PD. Factors associated with nursing assistant quality-of-life ratings for residents with dementia in long-term care facilities. Gerontologist. 2005;45 Spec No 1(1): 106-14.

53. Gräske J, Meyer S, Wolf-Ostermann K. Quality of life ratings in dementia care - a cross-sectional study to identify factors associated with proxy-ratings. Health Qual Life Outcomes. 2014;12(1):177.

54. Thorgrimsen L, Selwood A, Spector A, Royan L, de Madariaga Lopez M, Woods RT, Orrell M. Whose quality of life is it anyway? The validity and reliability of the quality of life-Alzheimer's disease (QoL-AD) scale. Alzheimer Dis Assoc Disord. 2003;17(4):201-8.

\section{Ready to submit your research? Choose BMC and benefit from:}

- fast, convenient online submission

- thorough peer review by experienced researchers in your field

- rapid publication on acceptance

- support for research data, including large and complex data types

- gold Open Access which fosters wider collaboration and increased citations

- maximum visibility for your research: over $100 \mathrm{M}$ website views per year

At BMC, research is always in progress.

Learn more biomedcentral.com/submissions 